\title{
Proposing ELA: Environmental Learning Algorithm for Enhancing Humans and Epigenetic Robotics Skills
}

\author{
Inderpal Singh \\ Department of Computer Science \&Engineering \\ CKD Institute of Management and Technology, Taran Taran, Punjab (India)
}

\begin{abstract}
Recently epigenetic robotics has been emerged as a new cognitive modeling approach in the field of artificial intelligence for modeling of autonomous mental development. The paper aims to develop new behavioral skills in the epigenetic robotics in different environment that would facilitate in enhancing the learning skills of humans utilizing machines. In an attempt to improve child mental development and growth, this revolutionary technology based upon artificial intelligence would be utilized for further development of machines that would behave like humans in a natural way. Utilizing the technique mentioned in the algorithm "ELA" (Environment Learning Algorithm), the humans will learn from machines and enhance their capabilities for better performance. The additional benefit for utilizing epigenetic robotics is to learn new skills autonomously through social interactions from different environments. The epigenetic robotics helps to remove the constraints on robotics that are already programmed pertaining to specifically task dependent.
\end{abstract}

\section{Keywords}

Natural intelligence, artificial intelligence, epigenetic robotics, skills, behavior, Robotics Social Interaction (RSI).

\section{INTRODUCTION}

Epigenetic Robotics [9], [23] belongs to interdisciplinary field and has a wide scope in the area of artificial intelligence. Different types of machine mechanisms, machine algorithms as well as machine structures are to be discussed in the area of artificial intelligence as example different types of mechanisms and algorithms are used in robotics for sensing and for their movement. These physical embodies of robotics generally uses several different-2 types of programs for performing separate types of functions by utilizing several programming constraints so that robotics will behave accordingly in future. In this paper, the major objective of authors is to utilize machine intelligence in different prospective and receives efficient results than earlier in the form of human natural intelligence. This idea gives a broader view to people how A.I Professionals are moving one step closer or one step forward to the human natural intelligence. One more important key point from authors prospective is: it is not a real natural intelligence as like human being intelligence but it is more than artificial intelligence so that's why they generally named "one step closer to natural intelligence". As authors are talking in general everybody knows human beings are the natural creatures of GOD. After the great advancement in the area of artificial intelligence and in other related fields those A.I Professionals, experts and scientists will not challenge our SUPREEME POWER/Nature. After studying from several books and some other sources author's chooses some of the important mechanisms for the design of special class of epigenetic robotics [9] whose main aim to provide open ended learning( that is a new type of learning from the existing environment) from our social environment through real time interactions. The purpose to authors study/ significance to study the special class of epigenetic robotics [9] is to improve or develop human's child mental growth [13], [21], [27]. In addition, the benefit to utilize this special class of epigenetic robotics [9] is to save human's time as well as energy on the time of training to their child's (or on the time of teaching to their kids) on their growing age or on their developing stage. Moreover, the additional benefit to utilize the special class of epigenetic robotics [9] is it gives company to their child's for playing time also and acts like as a friend that indicates these natural human beings may have machine friends. In short, natural human beings may also learn from the man-made artificially created machines. This new platform for open ended learning [14], [21] concept utilizes different developmental principles and mechanisms these physical embodies of robotics machines may behave accordingly on the time of providing training of their child's. The main advantage to utilize this new type of learning [14] is to facilitate people for the development of new skills in their new born babies (i.e. growing human infants) through machines like physical embodies[15] of epigenetic robotics[9][23]. As data collected by authors from their surveys, experts are collaborating their efforts with the different professional's related to different fields for developing new skills in human infants from the existing environment through social interaction with the physical embodies[15]. These physical embodies [15] help us for sharing their knowledge and information with the other human infants. The expectation of several experts from this recent advancement is to prepare upgraded machines for future generation so that they will in future behave like normal human infants (that is only behave like natural human beings they are actually not real human beings).

In addition, this large amount of contribution of epigenetic robotics [9],[23] in artificial intelligence also provides a collection of some distinct features as like "shows its autonomous learning behavior during real-time interactions" [12] with the environment. This real time interaction will only be possible through the embodied bodies [15] of epigenetic robotics [9]. In the absence of embodied bodies [15], the interaction between environment and epigenetic robotics [9],[23] is not possible. Before starting the interaction with the physical embodies [15], experts has need to study its basic structure that includes number of sensors and effectors [11] whose main function is to collect data or information from the environment [6] on the time of real time interaction with the environment while utilizing two different types of interactions viz. natural inter-personnel interactions \& social interactions [2]. By carrying one more additional unique feature [9],[23] of "feature of self-exploration" [29] the self-made brain structures [18] will be easily automatically build in physical embodies[15] of epigenetic robotics [9][23] by utilizing several principles and mechanisms. In addition, the best utilization of this unique feature [9][23], the class of 
epigenetic robotics [9],[23] will be easily implemented through path-following-method in future if experts realize its need for further alterations [4] in their physical embodies [15]. Even this method or approach will always not be appropriate in most of the cases but it may fail sometimes on that time when epigenetic robotics [9],[23] physical embodies [15] will not react like physical reality [21] (that is similar behavior as like physical reality). As authors collected data from their surveys and most importantly they listened some of the lecture sessions of experts on you tube during their conferences, they said epigenetic robotics [9],[23]is a big challenge for our scientific community now a days. They (Experts) want to create natural intelligence in physical embodied[15] machines [10] (that is epigenetic robotics [9][23]) as like human infants [15], [19] so that in future artificial machines will behave naturally as like normal humans beings [24]. By utilizing such type of machine intelligence autonomous child mental development [11], [26] will be easily enhanced and correspondingly they successfully adapt new skills from the continuously changing environment [8], [3].For implementing autonomous learning behavior in machines researchers took help from the different cognitive developmental mechanisms (CDM) [16] in physical embodied bodies[15] so that the improvement in their behavior should be come accurately ( that is according to the design of developmental mechanism) and on the another side simultaneously they uses hybrid approach for its effective utilization that can be diagrammatically represented as in figure.1:

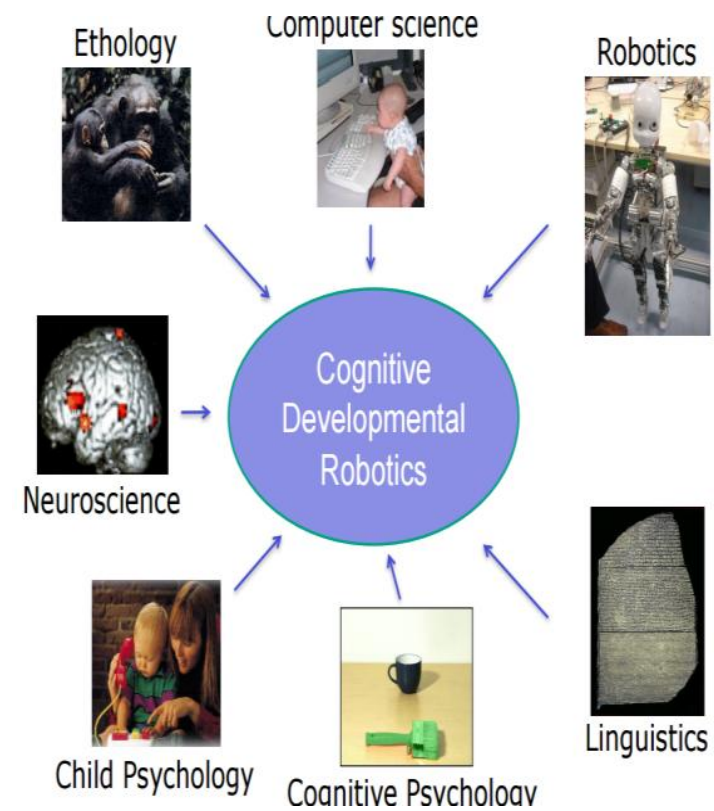

Fig.1: Cognitive Developmental Robotics - A Hybrid Approach [28].

The benefit to utilize this hybrid approach is its further use in several intrinsic learning principles for implementing autonomous learning behavior [5] in the class of epigenetic robotics [9],[23]. These learning principles along with its design issues give the complete description / or / understanding of the basic concept of human cognitive development process (HCDP). As authors saw several design issues are used in the class of epigenetic robotics [9] ,[23]but the two most common design issues are listed below:
$>$ Adapt dynamic environment firstly during the management of complex tasks.

$>$ Self-made brain structures [18] insider robotics brain that may further provide temporal Development Structure.

These design issues uses several synthetic and constructive approaches in CDP (Cognitive Development Process). The main function of CDP is to provide real time interactions with the body, brain as well as environment [13]. If at once a real time interaction with the environment through CDP is possible then automatically self-made brain structures [18] will be made in their physical embodies [15] and will work and behave accordingly (that is self-made brain structures [18]) . The main function of these self-made brain structures [18] is to store own knowledge base and user experience in their memory database [25] on the time of real time interactions with the environment. In addition, the class of epigenetic robotics [9],[23] may take one better advantage of the other classes of robotics viz. nano-robotics and toyrobotics etc. They may use as an agent to another classes of robotics that shows an epigenetic robotics [9],[23] act as master and the other class of robotics act as a slave ( that is for the same epigenetic robotics master). For implementing these self-made brain structures [18], neuroscientists generally use epigenetic robotics architecture (ERA) [17] that can be diagrammatically represented in fig.2:

\section{Epigenetic Robotics Architecture}

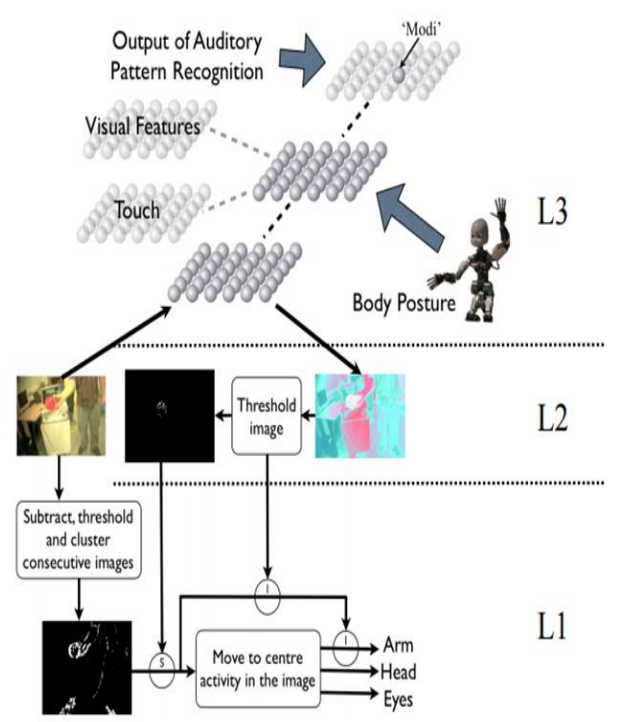

Fig.2: ERA- Epigenetic Robotics Architecture [28]

The motivation towards the utilization of ERA is to guide the modeling efforts to physical embodies [15] during their real time interaction [17] with the environment.

In this paper, authors designed a new methodology named "ELA" (Environment Learning Algorithm) for providing a new method for open ended learning[14] through machine intelligence for achieving better performance and results in future so that machines will behave naturally in future as like human infants[15],[19]. As authors noticed the main existing problem in the field of artificial intelligence is everything in robotics machines are programming based constraints as programmers and experts embedded programs in robotics 
machines that will behave accordingly presently. That clears robotics are now a days actually doing low skilled jobs as an example programming constraints put on machines robotics will behave accordingly and except that they will not do anything according to their wish as an example they do not have ability to think like humans or in other words you may say they will not work like humans wish etc. In this case, robotics acts as a slave and human act as a master. So, as per authors opinion this new designed methodology may help to remove several programming constraints [1], [7] on robotics machines and contributing their efforts for developing new skills in the class of epigenetic robotics [9],[23] by utilizing cognitive development principles (CDP) and learning mechanisms that may in future will facilitate our society people for self-driving their own innovation [22] through selfmade brain structures [18] in epigenetic robotics [9],[23]. The motivation towards the class of epigenetic robotics [9],[23] is also help to develop new behavioral skills in human infants or human kids by utilizing autonomous learning behavior in two different environments viz. real environment and virtual environment. As Authors studied this epigenetic robotics[9] ,[23]field has a great scope in future that's why till now scientists belongs to different areas are working hard or put great efforts for achieving maximum success in this field

\section{RESEARCH DESIGN}

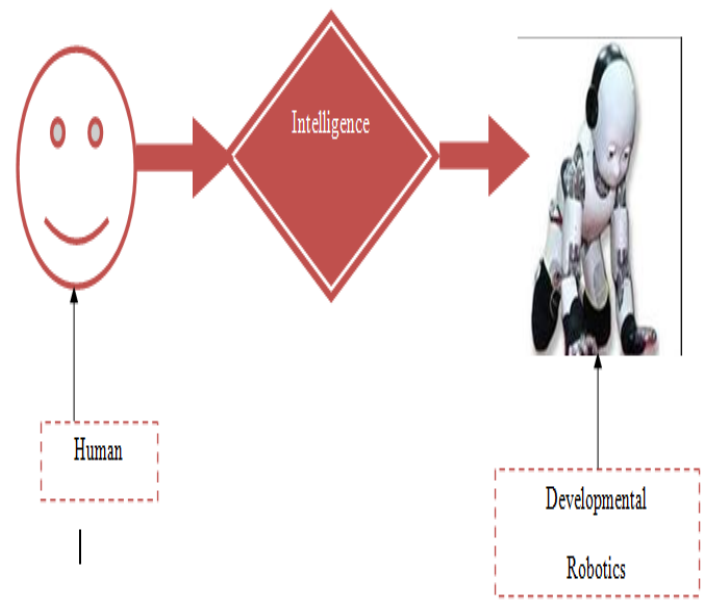

Figure.3:Intelligance act as an interface between human and epigenetic robotics.

\section{A ROADMAP-FOR INTELLIGENCE TRANSFORMATION MODE (ITM)}

This intelligence transformation mode tells us how machines will behave naturally as like humans infants. This new roadmap gives us a step closer to natural intelligence but actually it is not real natural intelligence- in which machines show their own natural (in artificial form) behavior or intelligence by utilizing self-deriving brain structures [18] in epigenetic robotics class [9],[23]. The diagrammatical representation of intelligence transformation mode can be diagrammatically shown in figure 4 :

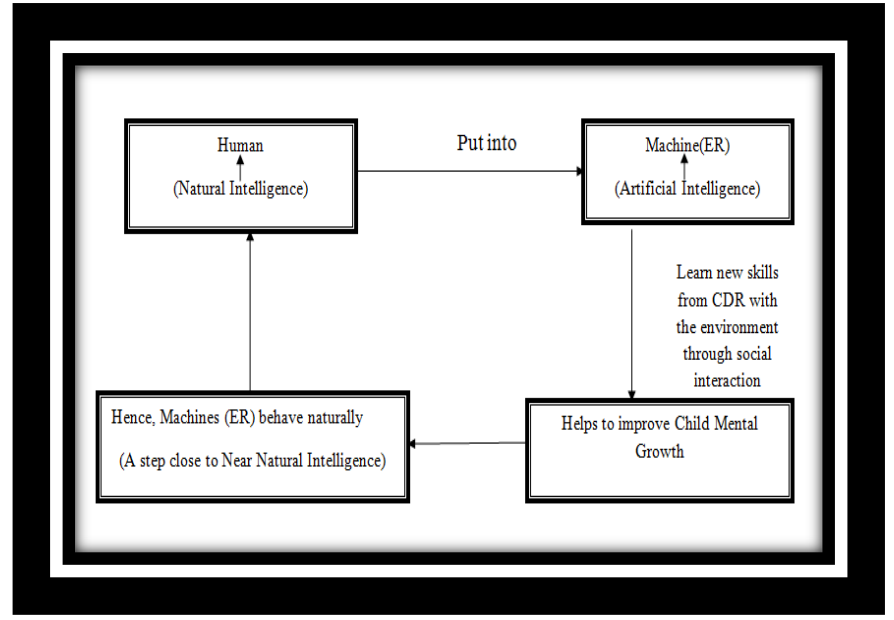

Figure.4: Flow of ITM (Intelligence Transformation Mode).

\section{PROPOSED ALGORITHM - ELA (ENVIRONMENT LEARNING ALGORITHM) \\ Step-1) Find an object (i.e. Human) inside the room.}

// through sensors and effectors ER can be easily detect the presence of humans at specified Location.

// (Object finding is based on the distance between the ER and object).

Object Finding: $=$ Distance $\mathrm{b} / \mathrm{w}$ ER + Actual Location of Object.

Step-2) IF Object inside the room is TRUE Then

Step-3) Check the status of Object \{In Motion (Moving), Motionless (Stationary)

Step-4) IF Object is Motion_Less then Go to Step 1

// Find another object inside the room.

Step-5) Else

Step-6) IF object is Moving (Object: $=$ MOTION_STATE) as like hand movements and legs movement then Epigenetic Robotics Monitor their actions through sensors \& effectors and record the whole movies in already embodied cameras in their physical body.

// the whole movie is record in ER self-made brain structure database.

Step-7) Store Everything in ER Memory. //

Database of ER.

Step-8) Go to step 1 and repeat from step 1 to step 7.

// for teaching more actions of humans to other Human Infants for their mental growth development.

Step-9) END IF UP TO STEP 4.

Step-10) Stop Learning.

Step-11) End. 
The benefit to utilize this new designed algorithm is to save human time as well as energy to teach or trained their kids while learning through their actions in the existing environment and correspondingly provide eco-friendly environment to their kids with machines. In this way, humans take a better advantage of epigenetic robotics [9],[23] for the development of their child mental growth [21],[27].

\section{CONCLUSIONS}

Authors concluded the epigenetic robotics [9],[23] is still in its infancy stage. This paper discussed about the different cognitive mechanisms used in epigenetic robotics architecture (ERA) along with its design issues. This designed methodology named "“ELA" (Environment Learning Algorithm) may help to enhance human infants or human kids learning skills from the existing environment and correspondingly by copying their actions as like hands and leg movements. In addition, epigenetic robotics [9] ,[23]may ask questions from human's kids by utilizing own self-deriving brain structures [18] in their physical embodies and correspondingly develop new skills autonomously learning new behavior through real time interactions in two separate types of environments viz. real or virtual. At the end, authors said cognitive mechanisms used in Epigenetic robotics [9],[23] plays a vital role for the improvement or development of child mental growth [13], [21], [27]. The success towards this field is demanding for the joint effort of many scientists that may belongs to different fields as like including computer scientists, neuroscientists, psychologists, philosophers and engineers.

\section{FUTURE SCOPE}

In future, this work will be extended by designing several new machine learning algorithms without having time bound constraints. By utilizing, these new designed machine learning algorithms researchers will easily learn more from the environment (i.e. by considering EVS given parameters as like temperature,humidity,pressure etc.) through Social Interactions with the class of Epigenetic Robotics (SICER). This may also help to develop new skills in epigenetic robotics [9], [23] as like human infants through self-learning from the environment.

\section{REFERENCES}

[1] Shen Qiang, Zhou Changle, Jiang Min, Meng Qinggang, Shang Changing, November 2014. A Developmental approach to robotics pointing via human robot interaction ,New Trend of computational intelligence in HRI, ELSEVIER.

[2] Breazeal Cynthia, 2009. Role of expressive behavior for robots that learn from people, Philosophical Transactions of the royal society.

[3] Cheng Gordon \& Atkeson Christopher, 2006. CoachingAn approach to efficiently \& intuitively create humanoid robot behaviors, IEEE.

[4] Simmons Reid, Forlizzi Jodi and Gockley Rachel, 2007. Natural Person following behavior for social robots, ACM, Virginia, USA.

[5] [Stoytchev. 2009. Some basic principles of developmental robotics, IEEE Computational Intelligence Society.

[6] Shen Min Wei \& Ranasinghe Nadeesha, 2008. Surprise based learning for developmental Robotics, ECSIS Symposium on Learning and adaptive behavior for robotics system, IEEE.

[7] Language Learning in children and robotics: - A Developmental Robotics Approach.

[8] Zeschel Arne.Saunders Joe \& Dautenhahn Kersin, September 2010. Integration of action and language knowledge: A Roadmap for developmental robotics, IEEE Transaction autonomous mental developmental.

[9] Talbott, 2015. Learning to perceive: A Developmental robotics approach to vision and object interaction, $\mathrm{PhD}$ Thesis, California.

[10] Bogdan Raducance, Cognitive DR: An intrinsic Motivation system to support social interactions.

[11] Thelen Esther, Sur Mriganka \& Stockman Ida, Autonomous Mental Development by robots and Animals, Access via science online site Pass.

[12] Abry Christian \& Schwartz Luc Jean, 2012. A Developmental robotics system for visual scene perception and language Acquisition, International program for robot cognition planning document, $\mathrm{PhD}$ Thesis, France.

[13] Schlesinger Matthew \& Cangelosi Angelo, Developmental Robotics: From babies to robots, Intelligent robotics and autonomous Agents, MIT Press.

[14] Konidaris. D George \& Stout Andrew, Intrinsically Motivated Reinforcement Learning: - A Promising framework for developmental robot learning, Cite seer.

[15] Hollich.J.George, Holder's Nathan \& Prince G.Christopher, Ongoing emergence- A Core Concept in epigenetic robotics, proceeding of the $5^{\text {th }}$ international workshop on epigenetic robot: Modeling cognitive development in robotic system.

[16] Bard Kim, An epigenetic approach aids the study of primate social cognition, proceeding of the $9^{\text {th }}$ international conference on epigenetic robotics: Modeling cognitive development in robotics system, UK.

[17] Cangelosi Angelo and Belpeame Tone, December 2010. Epigenetic Robotics Architecture, IEEE Transactions on autonomous mental development, USA.

[18] Weng Juyang, 2004. Developmental robotics - Theory and experiments, Embodied intelligence laboratoryInternational journal of humanoid robotics, World Scientific publishing company.

[19] Philibert A.Robert, beach H.R Steven \& Dogan V.Meeshanthini, Current and future Prospects for epigenetic biomarkers of substance use disorders, USA.

[20] Epigenetic Marks by foundations for a child's future abilities, University of Southampton, 2015.

[21] Bell .J Anthony, 2013. Levels and Loops: The future of Artificial Intelligence, The royal Society, USA.

[22] www.gereports.com/jim-lawton-when-humans-androbots-work-together/

[23] https://mitpress.mit.edu/books/developmental-robotics.

[24] a-labs.aldebaram.com/science-blog/featuredresearch/developmental-robotics.

[25] whatis.techtarget.com/definition/developmental-robotics. 
International Journal of Computer Applications (0975 - 8887)

Volume 180 - No.8, December 2017

[26] nivea.psycho.univpariss.fr/FeelingSupplements/CurrentA utonomousRobotics.htm.

[27] hys.org/news/2015-06-tool-standards-epigenetic.html.
[28] http://shanghailectures.org/sites/default/files/lectures_sli des/Cangelosi-ShanghAI2014.pdf

[29] https://en.wikipedia.org/wiki/Developmental_robotics. 